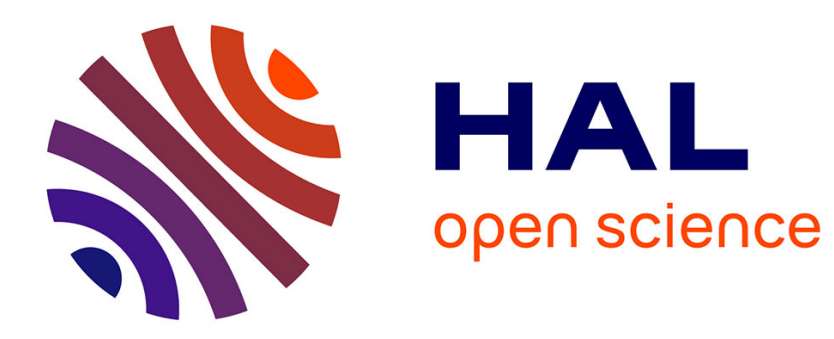

\title{
Le réseau technique est-il un impensé du XVIIIe siècle: le cas de la poste aux chevaux.
}

\author{
Nicolas Verdier
}

\section{To cite this version:}

Nicolas Verdier. Le réseau technique est-il un impensé du XVIIIe siècle : le cas de la poste aux chevaux..

Flux - Cahiers scientifiques internationaux Réseaux et territoires, 2007, 68, pp.7-21. halshs-00170049

\section{HAL Id: halshs-00170049 \\ https://shs.hal.science/halshs-00170049}

Submitted on 6 Sep 2007

HAL is a multi-disciplinary open access archive for the deposit and dissemination of scientific research documents, whether they are published or not. The documents may come from teaching and research institutions in France or abroad, or from public or private research centers.
L'archive ouverte pluridisciplinaire HAL, est destinée au dépôt et à la diffusion de documents scientifiques de niveau recherche, publiés ou non, émanant des établissements d'enseignement et de recherche français ou étrangers, des laboratoires publics ou privés. 


\title{
Le réseau technique est-il un impensé du XVIIIe siècle : le cas de la poste aux chevaux ${ }^{1}$.
}

\author{
Nicolas Verdier CNRS \\ UMR 8504 Géographie-Cités
}

Pour les spécialistes des réseaux techniques, les premiers résultats des travaux du groupe réseaux, au milieu des années 1980, représentent une avancée incontestable, si on les juge à l'aune des propositions de Maurice-François Rouge (toujours cités comme étant le premier à s'interroger sur les réseaux dans le cadre des sciences sociales), voire si on le compare aux propositions plus élaborées mais passées inaperçues en dehors de la géographie de l'époque, de Max Sorre. Dans les faits cette période, qui court de l'immédiat après-guerre jusqu'au milieu des années 1950 est un moment riche en questionnements sur le réseau. Il suffit de renvoyer sur ce point aux travaux de Pierre Monbeig en 1952, voire à ceux de Jean Gottmann en 1947, ou encore à ceux de Rouge en 1947 et 1944². Mais, à cause de changements profonds à l'intérieur de la discipline géographique, ceux-ci ne se sont pas imposés aux auteurs du milieu des années 1980. Autrement dit, ces premières recherches du Groupe Réseau, en s'attribuant un ancêtre bien spécifique et en en oubliant d'autres oriente les conceptions de ses chercheurs. Chaque groupe de recherche, chaque revue, chaque discipline procède de cette façon qui n'est pas, en-soi critiquable, mais qu'il est nécessaire de conserver en mémoire pour comprendre les orientations de cette recherche.

Dans le cas du Groupe Réseau cette recherche-choix d'ancêtres s'est effectué à au moins deux niveaux : tout d'abord celui des recherches proches (les travaux depuis une quarantaine d'années), ensuite celui des utilisations historiques du concept de réseau, et plus précisément une exploration du moment de la fabrication du concept. Ce second niveau s'explique en grande partie par l'impulsion produite par la rencontre entre les recherches de

\footnotetext{
${ }^{1}$ Cette recherche fait suite à une première étude menée grâce à un financement ATIP du CNRS : VERDIER N. (dir.), Territoire et communications : Évolution des conceptions et des mesures dans les réformes du tarif postal, 1759-1848, 2002-2004.

2 Rouge, Maurice-François, "L'organisation de l'espace et les « réseaux»", Hommage à Lucien Febvre. Éventail de l'histoire vivante, offert par l'amitié d'historiens, linguistes, géographes, économistes, sociologues, ethnologues, Paris : Armand Colin, 1953, pp. 401-405. SORRE, Maximillien, Les fondements de la géographie humaine, Paris, Armand Colin, 1950; MonBeIG, Pierre, Pionniers et planteurs de Sao Paolo, Paris, Armand Colin, 1952 ; GotTMAnN, Jean, "De la méthode d'analyse en géographie humaine, Annales de géographie, 1947, pp. 1-12. Rouge, Maurice-François, "Vers une nouvelle discipline. L'organisation de l'espace", Mélanges d'histoire sociale, 1947, vol. V, pp. 45-54; du même auteur, La géonomie ou l'organisation de l'espace (Paris, Librairie générale de l'institut d'urbansime de Paris (droit et jurisprudence), et en 1951.

De nombreux textes sur ces questions m'ont été signalés par Jean-Louis TISSIER, je profite de cette occasion pour l'en remercier.
} 
Gabriel Dupuy pour la France ${ }^{3}$, et celle de Joël Tarr pour les Etats-Unis ${ }^{4}$, cela sachant que pour ce dernier la recherche en histoire des techniques et des sciences est une relative évidence dans le champ institutionnel américain. On doit évoquer ici les programmes de la National Science Foundation qui financent non seulement des recherches en sciences et en technologies, mais aussi en histoire et en philosophie des sciences, depuis sa création, en 1950. Au-delà de cette rencontre entre deux chercheurs qui forme probablement l'un des éléments déclencheurs, c'est plus largement au contexte de la fin des années 1970 et du début des années 1980 qu'il faut renvoyer. La discipline historique connaît alors une phase d'expansion tant dans ses objets que dans sa réception dans la société qui la place en position de force dans les réflexions naissantes. C'est ainsi presque sans justification qu'une demijournée d'étude ayant pour thème "Émergence du concept de réseau territorial" se déroule en juin 1986. Cette relation entre sciences de l'ingénieur et sciences sociales, un temps forte, se réduit dans les années qui suivent. Conséquemment, ses résultats sont les références primordiales - lorsque ce ne sont pas les seuls —, pour les héritiers de cette période fondatrice, lorsqu'ils se penchent sur la généalogie de leur objet.

Or, l'un des résultats de ces premières recherches ancre nettement le concept de réseau technique territorial du côté des impensés du XVIIIe siècle, et des inventions du début du XIXe siècle. Cela même si de nombreux objets techniques, comme les chemins de fer tardent à employer le concept forgé à l'école polytechnique dans les années 1820-1830. Trois textes de 1986, pour certains réédités sous différentes formes, composent l'essentiel de la démonstration ${ }^{5}$, en partie consolidée par un rapport de recherche très documenté en $1988^{6}$. Chacun fournit une définition — souvent très stricte — du réseau et insiste, soit sur la hiérarchie, soit sur l'interconnexion, soit l'absence du mot, soit encore sur l'absence de réflexion à une échelle assez petite pour que le concept n'apparaisse avant le premier quart du XIXe siècle. La mise en série de ces textes, qui peuvent cependant être décrits par l'aspect restreint de leur objet, laisse entendre qu'avant les années 1820 il est peu probable de trouver l'usage du concept de réseau. D'autres textes, comme celui de Marcel Roncayolo sur Michel

\footnotetext{
${ }^{3}$ DupuY G., 1977. Urbanisme et Technique, chronique d'un mariage de raison, Thèse de l'Université Paris V.

${ }^{4}$ TARR J. 1978. The Impact of Transportation Innovation on Changing Spatial Patterns: Pittsburgh, 1850-1934, Essays in Public Works History, Essay No. 6, Public Works Historical Society, Chicago.

${ }^{5}$ LEPETIT B., 1986. «L'impensable réseau, les routes françaises avant les chemins de fer », Cahiers du Groupe Réseau, $\mathrm{n}^{\circ}$ 5, pp. 12-29. GuILlERME A., 1986. «L'émergence du concept de réseau 1820-1830 », Cahiers... op. cit., pp. 30-47. RiBeILl, G., “Au temps de la Révolution ferroviaire, l'utopique réseau”, Cahiers... op. cit., pp. 51-66.

${ }^{6}$ Guillerme A., 1988, Genèse du concept de réseau 1760-1815. Territoire et Génie en Europe de l'ouest, Rapport pour le compte du Ministère de l'équipement et du logement. Délégation à la recherche et à l'innovation. (IFU Jh GUI).
} 
Chevalier, en 1989 abondent, à juste titre, dans le sens de l'usage du concept chez les ingénieurs polytechniciens et Saint-simoniens ${ }^{7}$. L'idée de cette naissance dans le premier tiers du XIXe siècle semble s'être relativement imposée depuis et les recherches sur des réseaux avant le réseau des années 1820 se placent plutôt dans une logique d'hapax que dans celle d'un savoir peut-être plus étendu dans la société ${ }^{8}$.

Une relecture minutieuse des textes invite cependant à en limiter la portée et à s'interroger de nouveau sur les usages possibles du concept de réseau avant le XIXe siècle. Par réseau, nous entendrons non seulement ici les éléments matériels minimums (un ensemble de lignes et de points, soit un tracé physique), mais encore une volonté organisatrice et gestionnaire qui dépasse en l'amplifiant le simple réseau physique par l'articulation volontaire des lignes et des points afin d'en faire un système. Il est incontestable qu'on ne trouvera pas le mot réseau dans les archives des institutions liées aux réseaux techniques territoriaux ${ }^{9}$. Mais, l'absence de mot, si elle handicape la pensée n'empêche pas l'existence d'autres formes de discours, graphique par exemple. Il semble par ailleurs tout à fait juste que le corps des Ponts et Chaussées ne s'appuie pas sur ce concept dans le cadre de son intervention sur le territoire français, qui se fait à grande échelle. La question de l'interconnexion, qui exige que toutes les branches d'un même réseau se rejoignent au moins en un point, posera longtemps des problèmes. La rupture de charges à Paris dans le cas du réseau ferroviaire est certaine. La médiocre hiérarchisation des lignes des systèmes de communication semble également généralisée avant le XIXe siècle ; soit dit en passant, ce critère semble perdre aujourd'hui de sa nécessité dans le cadre du Web et des échanges P2P. L'évidence de la nécessité d'une hiérarchie a laissé place en vingt ans à des pratiques alors imprévisibles. Ce décalage d'une vingtaine d'années qui montre combien les conceptions ont pu évoluer en s'enrichissant nous incite à opérer un autre déplacement, en aval celui-ci, à la recherche d'usages et de concepts probablement difficiles à percevoir dans les postures des années 1980, et qui offriront autant de nouveaux modèles d'intelligibilité des réseaux.

\section{L’absence du mot : la représentation graphique}

\footnotetext{
7 RoncAyolo M., 1989. "L'aménagement du territoire (XVIIIe-XXe siècle)", in A. Burguière et J. Revel, Histoire de la France, L'espace français, Paris, Seuil, 1989, pp. 510-643, et plus particulièrement, "Le temps des essais : techniques et représentations du territoire", pp. 532-542.

${ }^{8}$ On verra de ce point de vue le très intéressant article de LETONTURIER E., 1996. "Le réseau mis en œuvre : le Rêve de Diderot", Flux, n²4, avril-juin, pp. 5-19.

${ }^{9}$ Notons cependant qu'André Guillerme en trouve des usages chez les spécialistes du Génie, comme d'Allent, dès 1802. Sur ce point GUILLERME A., Genèse du concept de réseau ... op.cit. p. 8 et pp. 142-143.
} 
Partons d'un texte connu, cité à l'envi - mais rarement lu dans son entier-, celui de D’Alembert dans le "Discours préliminaire" de L'Encyclopédie en 1757. Le texte nous intéressera sous plusieurs angles, mais limitons-nous pour l'instant à son rapport à la représentation graphique et plus précisément cartographique.

“L'ordre encyclopédique général sera comme une mappemonde ${ }^{10}$ où l'on ne rencontrera que les grandes régions; les ordres particuliers, comme des cartes particulières de royaumes, de provinces, de contrées ; le dictionnaire, comme l'histoire géographique et détaillée de tous les lieux, la topographie générale et raisonnée de ce que nous connaissons dans le monde intelligible et dans le monde visible ; et les renvois serviront d'itinéraires dans ces deux mondes, dont le visible peut être regardé comme l'Ancien, et l'intelligible comme le Nouveau."11

Ce que D'Alembert effectue, dans ce court extrait c'est dépasser l'aspect finalement classique d'une représentation arborescente des savoirs à l'époque - on la trouve depuis très longtemps, dans les cosmographies par exemple - pour montrer que L'Encyclopédie si elle est lisible sous cette forme l'est aussi sous une autre. C'est le rôle du renvoi qui est décrit comme un autre cheminement possible du raisonnement. Ce cheminement, cet itinéraire intellectuel, peut se lire métaphoriquement sur des cartes qui croisent les deux modes de lecture. On a donc une géographie des savoirs qui articule arborescence et itinéraires ; soit un ensemble qui, pour être pensé, s'appuie sur la carte. Prenons ce texte au sérieux : il montre bien que l'absence d'usage du mot n'empêche pas une conception complexe qui articule des lignes (renvois) et des points (définitions) dans un ensemble formant un système de savoirs (L'Encyclopédie). Mais il montre aussi qu'une façon de dire cela passe par une représentation graphique.

Travaillant sur la Poste aux chevaux et sur la Poste aux lettres, et plus généralement sur les voies de communication depuis quelques années il me semble possible de mobiliser ici quelques exemples qui montreront des usages anciens de ce type de conception dans une telle institution $^{12}$. Les routes de Poste ont en effet une particularité pour les historiens de la cartographie : elles sont le premier objet à avoir été représenté dans le cadre de la cartographie thématique - entendons par là une cartographie qui dépasse la simple localisation pour représenter une autre information quelle qu'elle soit. Dès 1632 Melchior Tavernier et Nicolas

\footnotetext{
${ }^{10}$ Diderot emploie déjà le mot dans le prospectus de l'Encyclopédie paru en 1750.

${ }^{11}$ D'AlemBert, "Discours préliminaires", de l'Encyclopédie. Sur cette question, on lira : LETONTURIER E., 1996. "Le réseau mis en œuvre : le Rêve de Diderot... op. cit.

${ }^{12}$ Sur cette enquête on verra : Bretagnolle, A. et Verdier N., "Images d'un réseau en évolution, les routes de poste dans la France préindustrielle (XVIIe siècle - début XIXe siècle)”, Mappemonde, n $79-3$, 2005: http://mappemonde.mgm.fr/num7/articles/art05301.html.
} 
Sanson publient une Carte Géographicque des Postes qui traversent la France (régulièrement rééditée ensuite). Comme l'ont montré Guy Arbellot puis Gilles Palsky, cette carte est la première à représenter un ensemble de routes sur le territoire du pays. Aucun géographe ne s'y était risqué jusque-là ${ }^{13}$. La seconde carte thématique recensée est due à Nicolas Sanson. Elle représente ce que nous appellerions aujourd'hui le réseau hydrographique français ${ }^{14}$. Le cartouche de la carte précise "J'ai tracé dans cette carte leurs cours (des rivières), leurs rencontres et remarqué leurs noms...”. On a donc là un ensemble de lignes et de carrefours représenté graphiquement.

\section{TAVernier M. et SAnson N., 1634 (2 ${ }^{\text {nde }}$ édition). Carte Géographicque des Postes qui traversent la France (document : Bibliothèque du Musée de la Poste ${ }^{15}$ )}

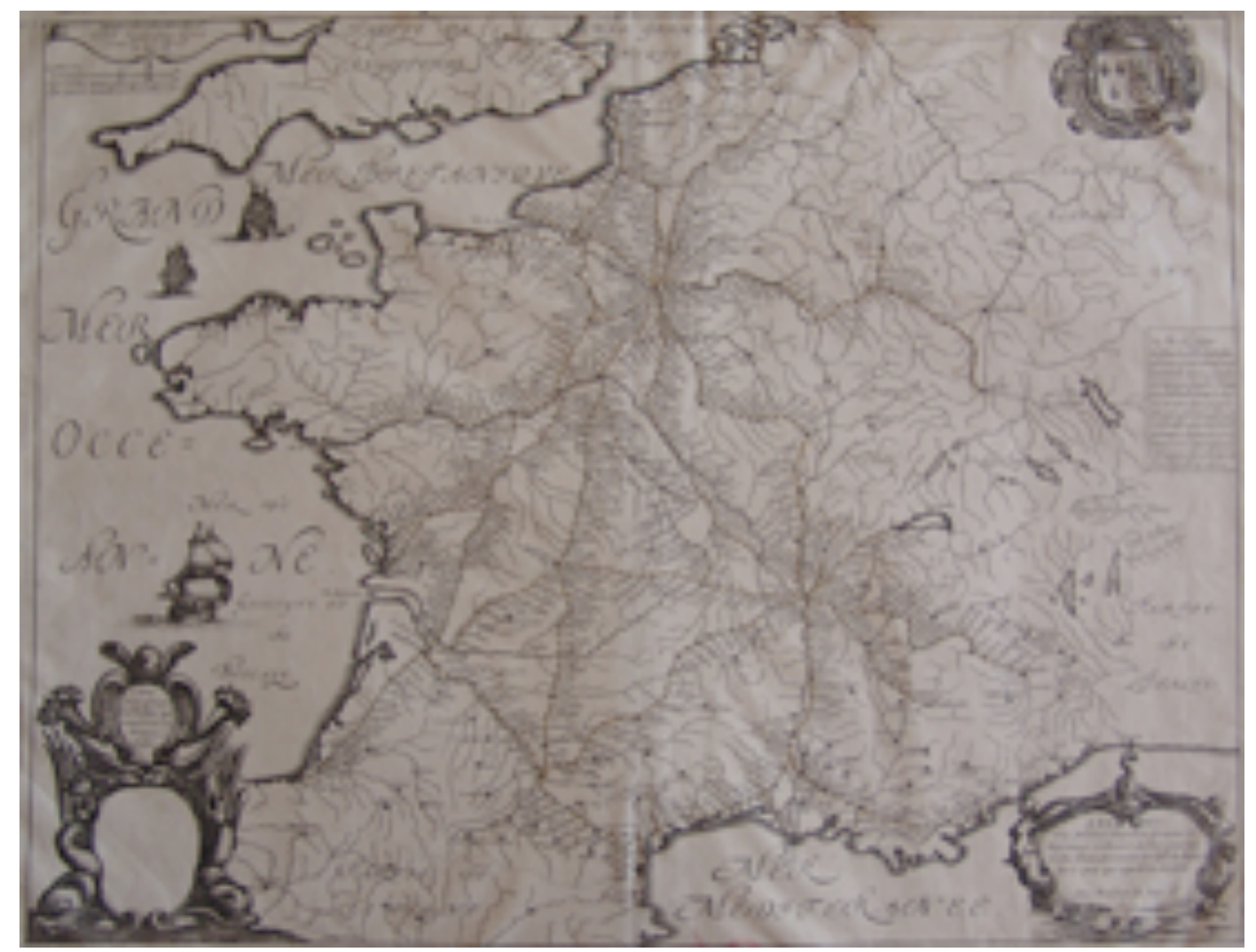

\footnotetext{
${ }^{13}$ TAVERnier M. et SANSON N., 1632. Carte Géographicque des Postes qui traversent la France; ARBELLOT G., 1980. "Le réseau des routes de Poste, objet des premières cartes thématiques de la France Moderne », $104^{e}$ Congrès national des Sociétés savantes, Bordeaux 1979, Hist. Mod., Paris, Bibliothèque nationale, pp. 97-115; PALSKY G., 1996. Des chiffres et des cartes, la cartographie quantitative au XIXe siècle, Paris, CTHS, pp. 1727.

${ }^{14}$ SANSON N., 1631. Carte des rivières de France curieusement recherchée. On notera par ailleurs qu'à une autre échelle les travaux sur les conduites des eaux des fontaines publiques de Paris par Nicolas de Fer en 1716 proposent également une représentation arborescente sur le plan (Sur ce point GUILlERME A., Genèse du concept de réseau ... op. cit.). Nicolas de Fer produira par ailleurs des cartes de la Poste aux chevaux.

${ }^{15} \mathrm{Je}$ tiens à remercier la bibliothèque du Musée de la Poste pour avoir autorisé ces photographies et leur diffusion. Mes remerciements vont également au personnel de cet établissement dont la compétence et la disponibilité ne sont jamais prises en défaut.
} 
Ces cartes n'auraient jamais été rééditées ni pillées par les copistes, on pourrait les ranger du côté des hapax, de l'idée tellement en avance sur son temps qu'elle n'avait pu être reçu dans la société. Mais tel n'est pas le cas. Le nombre des cartes de Poste va aller croissant, diffusant par là même une image de la forme de la France ${ }^{16}$. Après la période Nicolas Sanson, son fils, Guillaume va s'associer officiellement avec Alexis Hubert Jaillot vers 1690. Dès la fin des années 1680 Jaillot avait commencé à produire des cartes de Poste, lançant de ce fait la période Jaillot qui, passant de père en fils, dure un peu plus d'un siècle. À partir de 1706, de grandes cartes (jusqu'à 120 X 120), de faible diffusion, sont publiées annuellement, ce qui permet de suivre l'évolution de la morphologie du système des routes. À ces éditions de cartes, la famille Jaillot ajoute dès 1703 la publication annuelle des Livres de Poste qui donnent l'ensemble des itinéraires desservis par la Poste aux Chevaux. Après 1715 ces Livres... se voient dotés d'une petite carte de France (15X15) sur laquelle sont indiqués les routes de Poste. Ces ouvrages n'ont rien de décoratif : ce sont des objets techniques dédiés aux voyageurs de l'époque, à l'égale des Indicateurs de chemins de fer. Ils décrivent précisément les trajets entre les grandes villes en laissant au lecteur la charge de combiner les routes entre-elles, à l'aide de la carte, pour construire leurs itinéraires.

\section{Carte générale des postes de France, Jaillot $1716^{17}$. \\ (document : Bibliothèque du Musée de la Poste)}

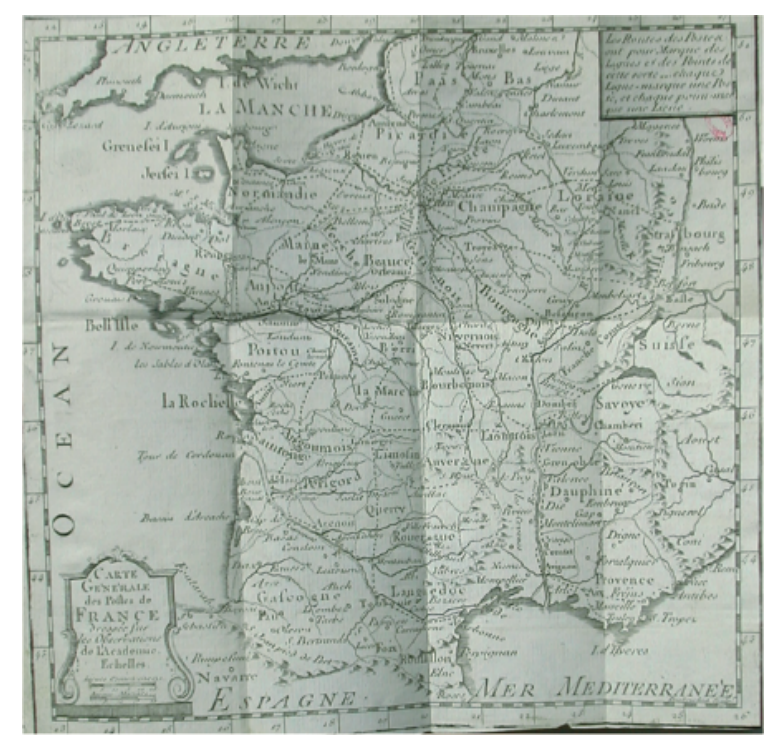

\footnotetext{
${ }^{16}$ ARBellot G., 1992. Autour des routes de Poste, les premières cartes routières de la France XVIIe-XIXe siècle, Paris, Bibliothèque nationale/Musée de la Poste.

${ }^{17}$ La carte présentée ici est plus tardive (1733) JAILLOT, 1733. « Carte générale des Postes de France », in Liste générale des Postes de France..., Paris, Chez le sieur Jaillot, Géographe ordinaire du Roy, 1733.
} 
À partir de 1771 les cartes sont plus grandes $(75 \times 75)$ et offrent une description fine de l'ensemble des voies de communication. Comme les livres de Poste, elles sont remises à jour tous les ans et offrent donc une information à jour sur le système des voies.

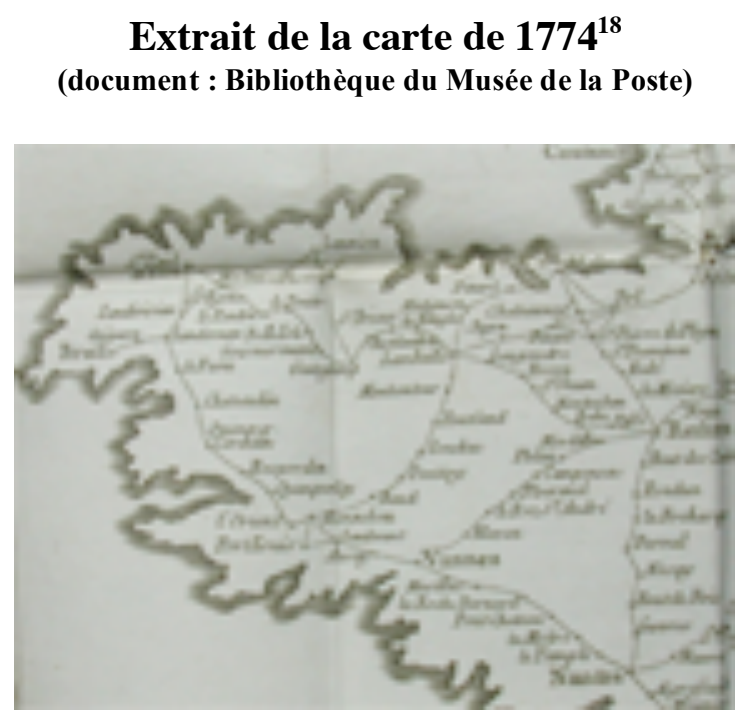

On a donc, à partir de la fin du XVIIe siècle une prolifération de cartes sur le système des voies de communication de la Poste aux Chevaux, qui s'accompagne à partir de 1716 de la mise en relation entre listes d'itinéraires et représentations cartographiques. Le fait de réunir dans un même ouvrage deux représentations d'un même système articulant relais de Poste et routes de Poste semble suffisant pour affirmer qu'il y a là, de façon précoce une pensée du réseau. L'utilité de ces cartes aux yeux du pouvoir est avérée par la mise en place d'un privilège royal d'édition de la carte officielle des postes au profit de la famille Jaillot. Leur utilité aux yeux du public semble évidente lorsque l'on note la présence de publications de cartes concurrentes malgré le privilège (Routes des Postes du Royaume de France de Nicolas de Fer (1700-1728, 1761), Carte du Royaume de France où sont tracées exactement les Routes de Postes par Robert de Vaugondy (1758)). Il y a là incontestablement une demande à satisfaire.

Mais, en dehors de ce laboratoire du réseau qu'est la Poste aux chevaux est-il possible de trouver d'autres traces graphiques d'une pensée articulant ainsi lignes et points dans le cadre d'un système ? la réponse est clairement affirmative et nous en prendrons deux exemples parmi des réseaux techniques territoriaux. Le premier se situe du côté du système de

\footnotetext{
${ }^{18}$ 1774. Liste générale des Postes de France pour l'année 1774, Paris, s.1.n.d.
} 
fortification de la France. Chacun connaît aujourd'hui les travaux de Vauban ceux de Cormontaigne, et plus tard ceux de Fourcroy sur les fortifications de la France. Ceux-ci ont été précisément étudiés par André Guillerme ${ }^{19}$. Mais, le plus souvent leurs conceptions sont vues comme n'offrant que des prémices d'une pensée du réseau. Or la pensée des fortifications semble bien avoir dépassé ce stade entre la fin du XVIIe et 1758, et probablement entre 1729 et $1758^{20}$

Échelle géographique très facile pour Sçavoir Promptement la Distance qu'il y a de l'une à l'autre des Principales Villes et Forteresses situées dans le Royaume de France, dans la Savoye, sur le Rhein, en Flandres et la Hollande dressé par le Sr Fauret ingénieur et géographe du Roy.

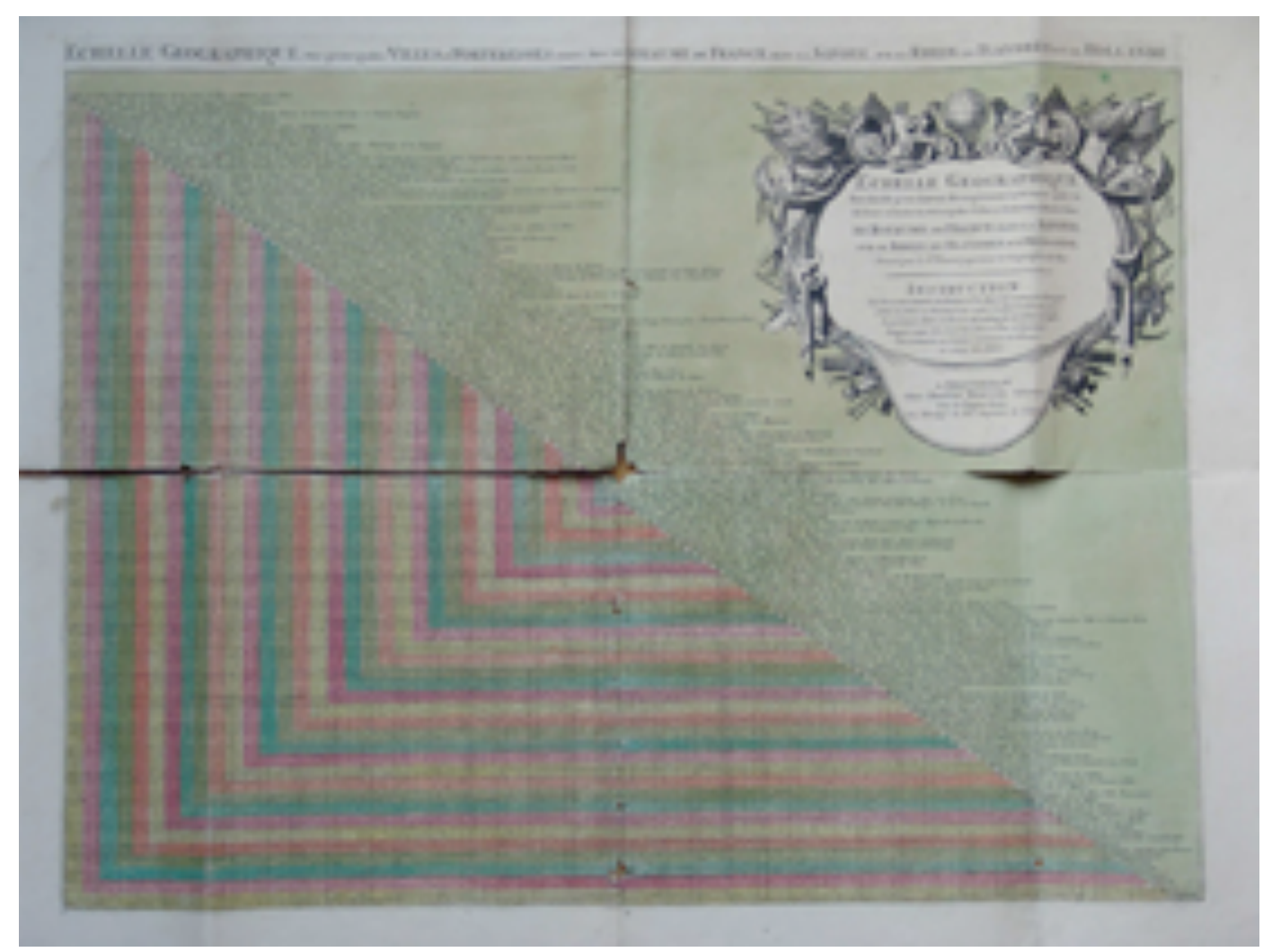

\footnotetext{
${ }^{19}$ Guillerme A., 1986. «L'émergence du concept de réseau 1820-1830», Cahiers... op. cit. et du même auteur: Genèse du concept de réseau ... op. cit.; FAUCherRe N. et Françors S., Places fortes bastion de pouvoir, Paris, Desclée de Brouwer, 1992.

${ }^{20}$ Le document, trouvé par Jean-Marc Besse, un lendemain de grande braderie à Lille n'a pas jusqu'ici pu être mieux identifié. Il est édité par Pierre Mortier à Amsterdam. On ne sait s'il s'agit du père ou du fils (ce dernier meurt en 1758). L'éditeur a publié en 1702 un texte de l'Abbé Du Fay sur les fortifications de Vauban, mais a surtout publié une Galerie Agréable du monde en 16 volumes dont 6 sur la France (le dernier étant publié en 1729). L'ouvrage offre des descriptions de forteresses, et de villes. Je n'ai pu en consulter jusqu'ici que deux exemplaires incomplets. Je profite de cette occasion pour remercier Jean-Marc Besse de m'avoir fait part de sa trouvaille.
} 
Il s'agit ici d'une matrice triangulaire des distances qui peut avoir été élaborée à partir des cartes des routes de Poste ou qui peut l'avoir été en s'appuyant sur la Carte générale des étapes de France dont on connaît quatre exemplaires (1710, 1746, 1755, 1795). Il s'agit d'une carte liée au Génie militaire, et dont l'objet est de dépeindre les voies de communication à employer pour se rendre d'un point à un autre du territoire. Cette dernière ressemble dans son principe aux cartes des routes de Poste, même si le niveau de simplification est beaucoup plus élevé et que par ailleurs les lieux de forte nodalité postale, comme Paris, comptent ici moins de liens ${ }^{21}$. Partant d'un ensemble de routes, la matrice offre un ensemble de possibilités de liaison entre chaque lieu. Le système de relations est ici avéré même si dans ce type de représentation l'idée de carrefour disparaît. Ce modèle est également employé lors de la mise en place du nouveau tarif de la Poste aux Lettres, en 1791. Celui-ci permet de calculer le tarif d'acheminement de courrier de centre théorique de département à centre théorique de département.

\section{Extrait du "Tableau des distances et des tarifs par départements". (document : Bibliothèque du Musée de la Poste)}

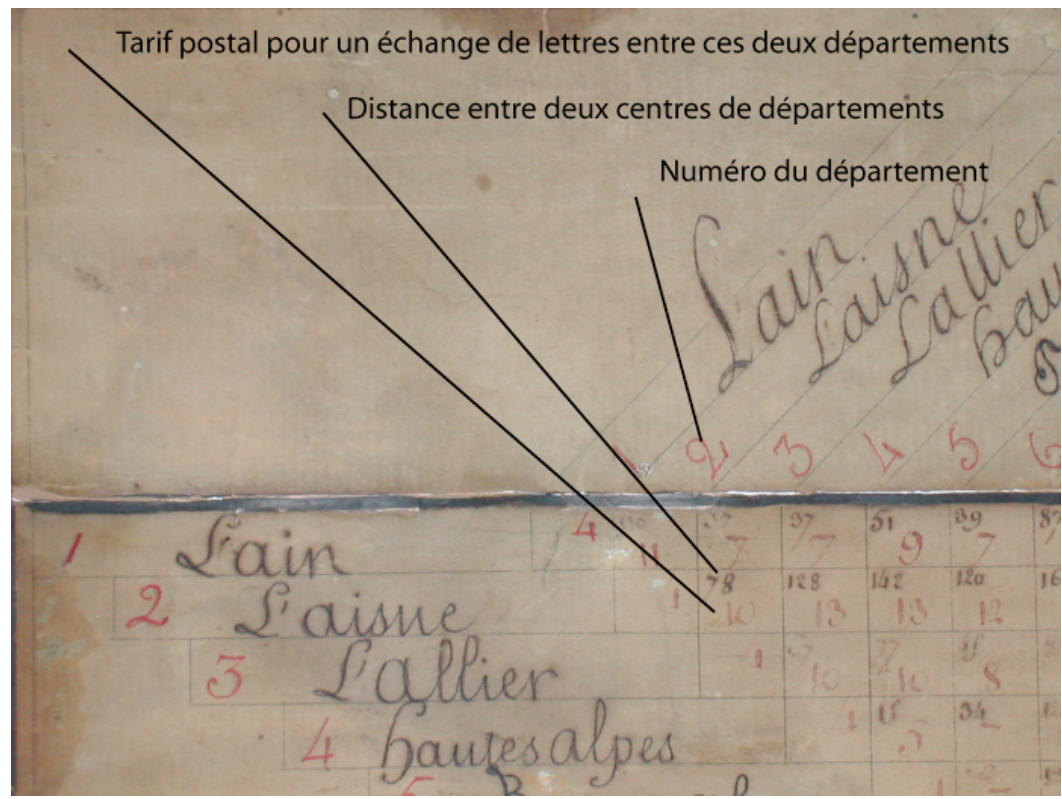

Même si dans ce dernier cas les distances sont toutes théoriques, puisqu'il s'agit à peu près des centroïdes des départements, ce qui importe ici est d'abord une pensée de mise

\footnotetext{
${ }^{21}$ Carte générale des étapes de France où sont tracées les Routes que tiennent les troupes dans leur marche, 1755, BNF Manuscrit 6435 F$^{\circ} 85$. Sur cette carte Konwitz, J. W., "Remplir la carte", in Cartes et figures de la Terre, Paris, Centre Georges Pompidou, 1980, pp. 304-315.
} 
en relation de l'ensemble des points entre eux par des lignes, qu'elles soient des routes militaires, ou des distances théoriques.

\section{L'absence du mot : un autre mot ?}

Ces objets graphiques, cartes et tableaux, voire les assemblages de ces formes de représentation ne s'appuient donc par sur le mot réseau. L'enquête sur les mots mérite cependant d'être poussée un peu plus avant en s'interrogeant sur l'utilisation possible d'autres mots. Dans ce dessein nous commencerons par évoquer le moment de l'invention de la théorie des graphes. C'est-à-dire une théorie visant à formaliser les rapports entre un ensemble d'objets et leurs relations. Les historiens des mathématiques s'entendent généralement pour faire de Leonhard Euler l'inventeur de la théorie des graphes, ou plus modestement de la topologie. Au-delà de cette appropriation qui mériterait certainement d'être interrogée, il semble intéressant de se demander quel est le vocabulaire employé par Euler. Chacun connaît le problème des Ponts de Koenigsberg : Étant donnée une île (A) entourée d'un fleuve qui se partage en deux bras, est-il possible de passer par tous les quartiers de la ville en empruntant tous les ponts une seule fois. Fondamentalement, il s'agit de déterminer le cheminement d'un point à un autre passant par une série de points intermédiaires. Le texte en Latin utilise les termes de viator (voyageur) et de transitus (trajet, passage). Nous sommes assez proches ici des problématiques d'organisation de la poste aux chevaux. Le mode de représentation de passage du fleuve est d'ailleurs très proche de celui employé par des cartes manuscrites de la Poste aux Chevaux à la fin du XVIIIe siècle.

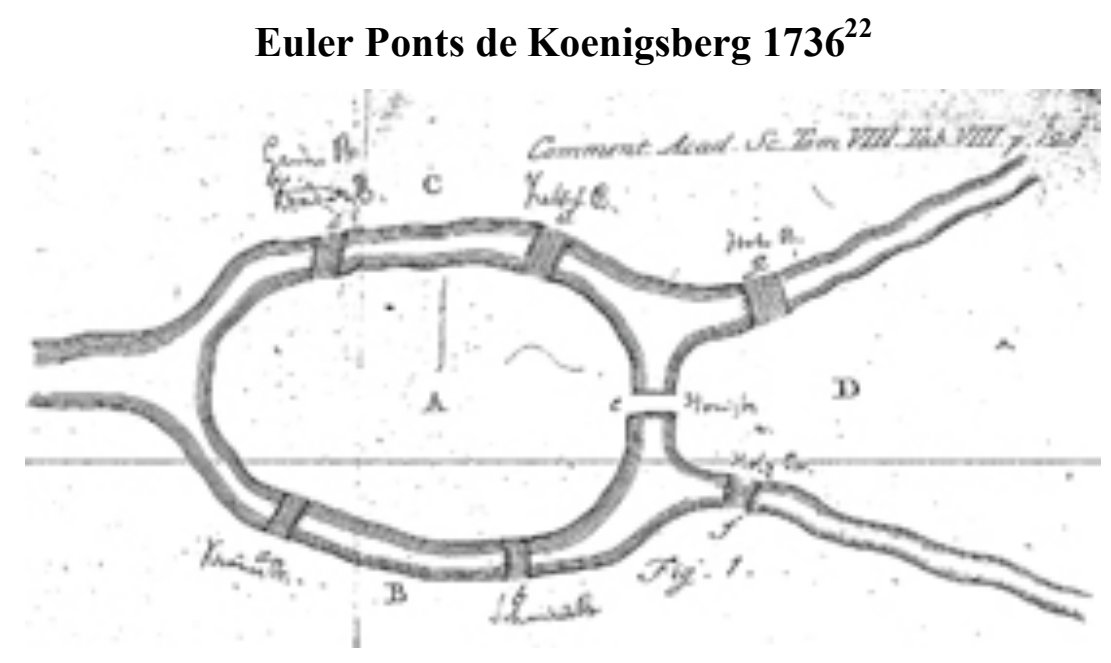

\footnotetext{
${ }^{22}$ EULER L., 1736. "Solutio problematis geometriam situs pertinentis", Commentarii Academiae Scientiarium Imperialis Petropolitanae, Saint-Petersbourg, vol. 8, pp. 128-140.
} 
Carte des Routes de Poste, 1688

(document : Bibliothèque du Musée de la Poste)

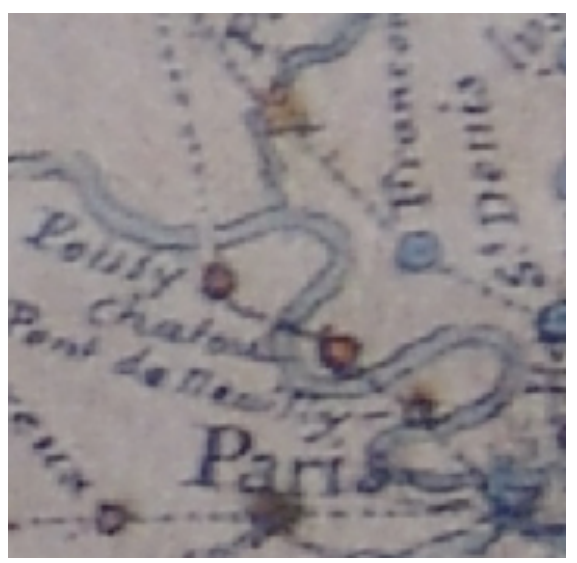

C'est donc sur le vocabulaire du voyage, ou plus modestement du déplacement, que s'appuie Euler, et c'est vingt ans plus tard sur le mot "itinéraire" que s'appuient Diderot en 1750 et D'Alembert en 1758 à propos de l'Encyclopédie ${ }^{23}$. Fondamentalement, la difficulté réside dans la relation entre ce vocabulaire du voyage qui sous-entend pour nous un usage orienté des voies de communication et le réseau, voire la théorie des graphes qui permettent de penser un ensemble des points et leurs relations. En 1740, lorsque Piganiol de la Force présente des "Cartes itinéraires de la France", il ne s'intéresse qu'à une série de cartes de détail qui se limitent au chemin parcouru ne tenant aucunement compte des carrefours ou embranchements.

Mais trente ans plus tard, Jean-Baptiste Ogée, un Ingénieur des Ponts et Chaussées, fait paraître un Atlas itinéraire de Bretagne contenant les Cartes particulières de tous les grands chemins de cette province. On est toujours du côté d'un vocabulaire du voyage, mais l'objet qu'il contient s'est complexifié. En dehors d'une carte générale qui présente l'ensemble des routes, les villes et lieux de passages importants, Ogée y présente des "Cartes particulières" qui offrent des choix de cheminements différents. Cette production peut correspondre dans les faits à une pratique récurrente des ingénieurs dans leurs circonscriptions : ceux-ci parcourent systématiquement les routes de leur ressort pour en connaître l'état. La pratique des ingénieurs des Ponts et Chaussées sur le terrain est donc potentiellement le moment d'une conceptualisation précoce sur le réseau.

\footnotetext{
${ }^{23}$ Diderot emploie le mot dans le prospectus de l'Encyclopédie paru en 1750, et nous avons vu plus haut que d'Alembert l'utilise dans le "Discours préliminaire".
} 
Piganiol de la Force, Nouveau voyage de la France avec un itinéraire et des cartes, Paris, Théodore Legras, 1740, p. 72 (document : Bibliothèque du Musée de la Poste)

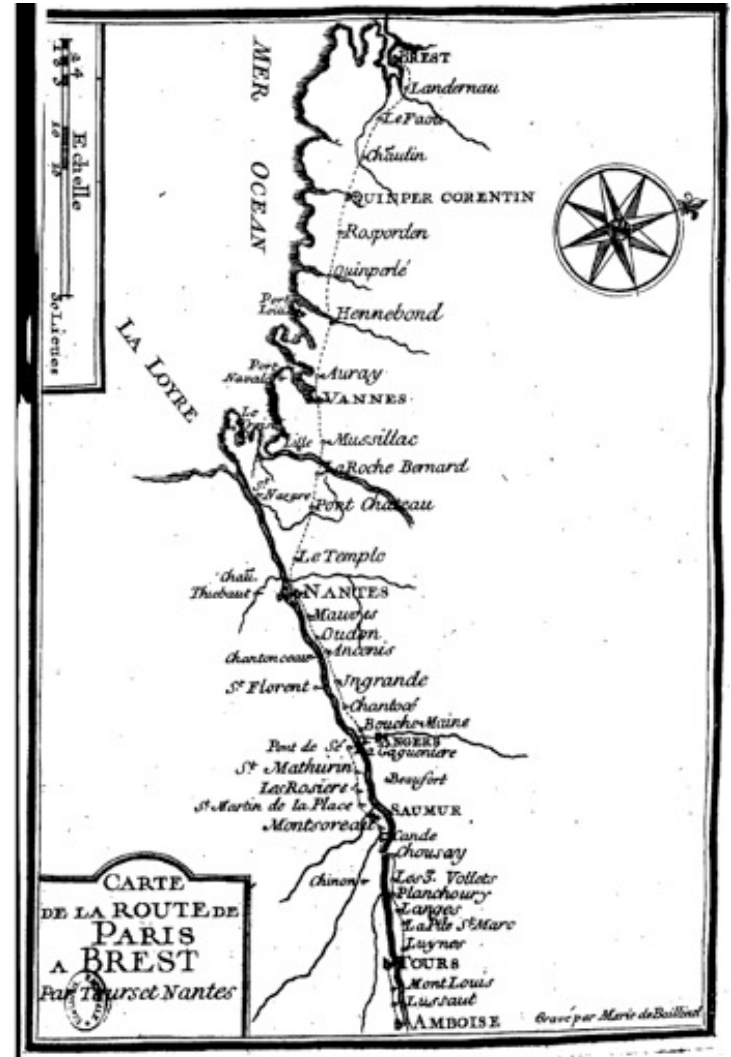

Planche VII de l'Atlas itinéraire de Bretagne... de Jean-Baptiste Ogée (1769).

(Collection de la Bibliothèque de l'Université de Caen)

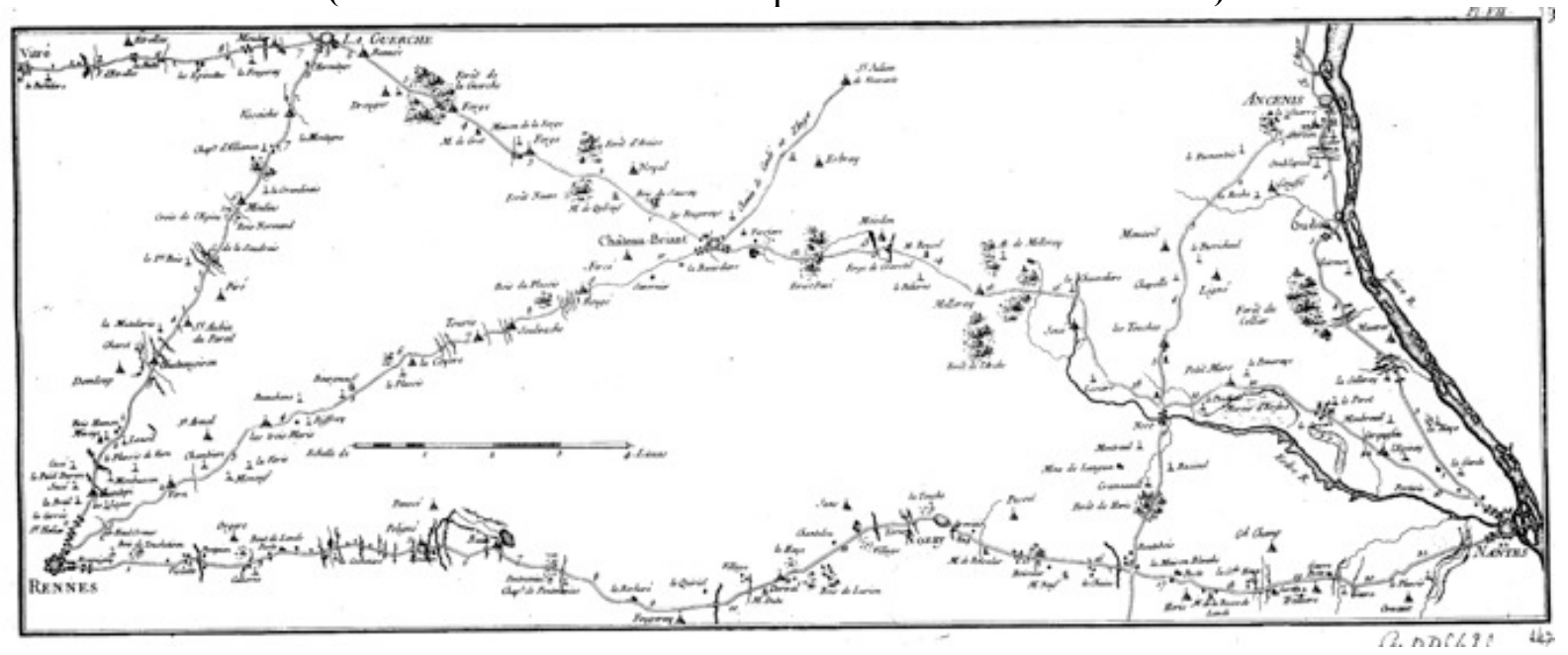

On notera ici que les carrefours importants, Nantes et Rennes, mais aussi Chateaubriand et La Guerche y sont représentés d'une façon différente des simples lieux de passage. La hiérarchie entre les villes joue sans aucun doute un rôle ici, mais elle ne suffit pas 
à expliquer le niveau de représentation de La Gueldre, voire de Chateaubriand. Il y a donc une mise en relation entre le niveau de nodalité observé et la représentation sur la carte. La représentation des voies de communication procède par une articulation de voies et de carrefours qui dépasse la simple contiguïté pour aboutir à un système.

Le deuxième tiers du XVIIIe siècle connaît donc un usage extensif du mot itinéraire propre à palier, en partie, l'absence du mot réseau. Mais alors que le mot "itinéraire” semble surchargé de sens à ce moment ${ }^{24}$ (comme actuellement le mot réseau) — il peut encore désigner les recueils de prières à dire lors de voyages - il s'appauvrit ensuite pour ne plus signifier que le chemin à suivre pour aller d'un point à un autre - comme dans l'Itinéraire de Paris à Jérusalem de Chateaubriand (1810), publié avec une carte, mais sans tracé de route. La mise en place du mot réseau coïncide avec cet appauvrissement ; même s'il ne s'agit que d'un médiocre indice, il s'ajoute aux précédents.

\section{La question de l'échelle}

L'échelle est-elle un critère pertinent pour penser le réseau? A priori non. L'argument qui consiste à affirmer que le corps des Ponts et chaussées ne pense pas le réseau parce qu'il n'a pas de carte des routes à l'échelle de la France au XVIIIe siècle n'a probablement pas de sens (ce que montre parfaitement le travail d'Ogée). La question est bien plutôt de savoir si la mission du corps des Ponts et Chaussées et de gérer un réseau, ou d'entretenir, voire de créer des tronçons à la demande de telle ou telle autorité, dont d'ailleurs la Poste aux Chevaux fait partie ${ }^{25}$. Il ne faut pas confondre ici les problématiques du corps des Ponts du début du XIXème siècle, armé de moyens et de pouvoirs (combien d'ingénieurs des Ponts sont alors députés ?), et celle d'un corps en cours de constitution, aux moyens limités qui soit, tente d'organiser les choses, comme le montre le Mémoire instructif sur la réparation des chemins du Contrôleur Général Orry en 1738, mais qui est d'abord là pour réparer 1'existant. C'est donc plus la question de la fonction du corps, que celle de l'appréhension à une certaine échelle qui pose problème. À cette date, les membres du corps se posent d'autres questions, et lorsqu'ils tentent d'y réfléchir, leurs projets sont peu applicables. Ainsi, comme l'a bien montré Bernard Lepetit, le texte d'Orry de 1738 offre une hiérarchisation des routes

\footnotetext{
${ }^{24}$ Les Atlas qui contiennent dans leur nom le mot itinéraire sont nombreux à partir des années 1760 . Brion de la Tour, Atlas itinéraire de l'Europe, adapté, quant à la France aux messageries royales, nouvelles diligences..., par le Sieur Brion, Paris, Brion, 1766 ; Le Rouge (trad.), Itinéraire de toutes les routes de l'Angleterre, Paris, Desnos, 1759, réédité en 1766. Puis vient la série des Indicateurs fidèles... de Desnos, publiés et republiés de 1764 à 1775 .

${ }^{25}$ On verra par exemple le cas du relais de Canon où l'on demande aux ingénieurs des Ponts et Chaussées le toisé et la mise en état des tronçons correspondants : M. G. Lesage, "La poste aux chevaux de Canon", Bulletin de la société des Antiquaires de Normandie, 1916, pp. 38-54.
} 
très précise. Son article 14 distingue les grandes routes qui mènent de Paris aux grands ports de mer et aux frontières, les routes de Paris aux autres capitales des provinces, les grands chemins, reliant les capitales de provinces entre-elles, les chemins royaux reliant les autres villes entre-elles, et enfin les chemins de traverse, allant de villes en villes sans poste ni messagerie. On notera l'aspect inapplicable de toute hiérarchie stricte de ce type, calée en même temps sur des destinations et des niveaux de ville. La route de Paris et Marseille est aussi celle qui va de Lyon à Marseille, voire celle de Dijon à Auxerre. L'échec du Mémoire... d'Ory, au moins en ce qui concerne la mise en place du réseau est dû en même temps aux tâches écrasantes de l'entretien routier, et à la fragilité de la réflexion. Cependant, il marque une étape sans laquelle les travaux d'Ogée n'aurait pu avoir lieu. Ce dernier dépasse les conceptions du Contrôleur Général en offrant une carte qui couvre la Bretagne. On y retrouve une hiérarchie urbaine en partie en phase avec le niveau de nodalité. Le niveau des voies n'est cependant pas différencié dans cet Atlas.

Ogée ingénieur des Ponts et Chaussées, Atlas itinéraire de Bretagne..., Nantes, Chez l'auteur, 1769 (Collection de la Bibliothèque de l'Université de Caen)

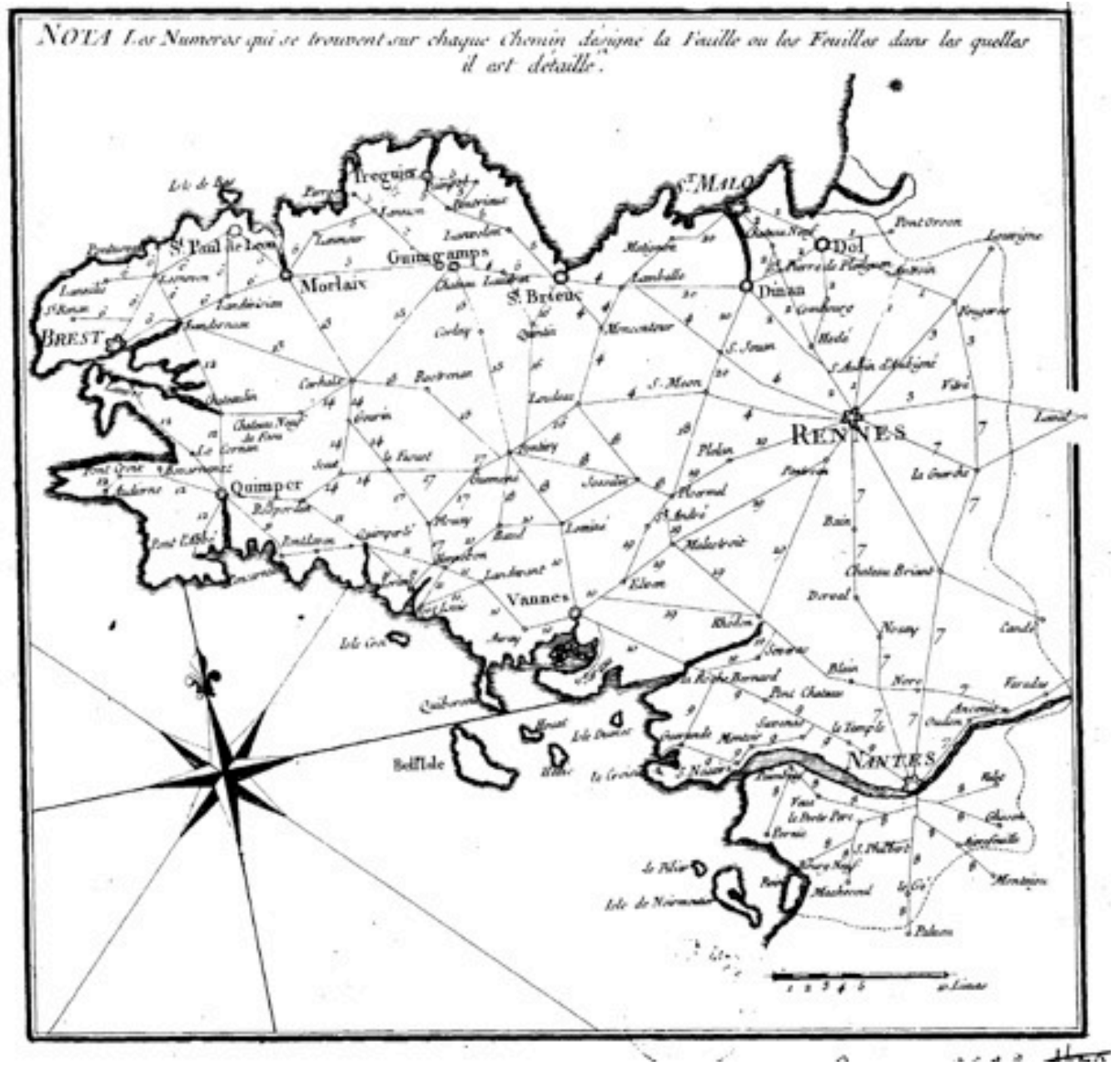


Ajoutons qu'à l'échelle du projet, qui se rapproche de celle de l'utopie (au sens premier du terme), ce qui peut apparaître dans la faiblesse de la réflexion sur l'applicabilité des conceptions, on trouve en $1781 \mathrm{chez}$ Achille Nicolas Isnard, une construction théorique qui lie niveau de ville, et niveau de voies de communication. Celui-ci articule routes et canaux, et en en percevant les conséquences sur l'organisation de l'espace :

“Les grandes routes devroient être multipliées de manière qu'elles formassent entre elles des figures triangulaires, des angles desquelles toutes les villes fussent le sommet. Il faut observer cependant de donner aux côtés de ces figures les sinuosités nécessaires à passer par les bourgs \& villages, lorsque les sinuosités ne sont pas telles que le transport en éprouve un allongement nuisisble $[\ldots]$.

Il seroit à désirer que la communication que nous venons de proposer de villes en villes, par des grandes routes, fût établie entre les centres de province par des canaux ou rivières navigables; le commerce intérieur de chaque province seroit servi par terre, \& les voyages de voituriers ne seroient jamais de long cours, le commerce de province à province seroit servi par eau." 26

On voit ici se profiler une organisation de l'espace en triangles, se rassemblant probablement en hexagones, qui rappelle les productions de Sea en $1811^{27}$, celles de Léon Lalanne $^{28}$ au XIXe siècle, puis celles de Walter Christaller au XXe siècle.

Enfin, pour en finir avec cette question d'échelle, s'appuyant en grande partie sur l'absence de cartes des routes à l'échelle de la France, nous avons montré, avec les cartes des postes (disponibles à la vente) ainsi qu'avec les cartes d'étape de l'armée (uniquement manuscrite) qu'il existe en France depuis le XVIIe siècle, et plus nettement encore depuis les années 1710, des représentations graphiques à l'échelle de la France qui permettent de penser l'articulation de points et de lignes dans le cadre d'un système de transport.

\section{L'interconnexion, la connectivité et la hiérarchie.}

La question de l'interconnexion est pour les chemins de fer particulièrement durable. Georges Ribeill a bien montré que la fameuse Étoile de Legrand (proposition de loi de février $1838)^{29}$ n'organise en rien un réseau connexe, mais qu'elle met plus modestement en place

\footnotetext{
${ }^{26}$ ISNARD, Achille Nicolas (Anon.), Traité des richesses, contenant l'analyse de l'usage des richesses en général $\&$ de leurs valeurs; les principes \& les loix naturelles de la circulation des richesses, de leur distribution, du commerce, de la circulation de monnoies \& de l'impôt..., Londres, François Grasset, 1781, 2 vol., vol. 1, pp. 113-116, la ciation est issue de la page 114.

${ }^{27}$ SEA, Mémoire sur les fortifications permanentes, Saint Petersbourg, 1811.

${ }^{28}$ Sur Léon Lalanne, on lira RoBIC, M.-C., "Cent ans avant Christaller... Une théorie des lieux centraux", L'espace géographique, 1982, pp. 5-12.

${ }^{29}$ Bretagnolles, A. et RoBIC, M.-C.,"Révolution des technologies de communication et représentations du monde», -1:«Monde-point et monde difforme»”, L'Information Géographique, juin 2005, vol. 69.
} 
une série de lignes convergeant vers Paris, mais ne s'y touchant pas. Paris est un lieu de double rupture de charge : voies de chemin de fer — rues ; rues — voies de chemin de fer. Il faut dans le cas de la Poste aux chevaux restreindre cet aspect de rupture de charge : Paris a un seul relais (en 1758 par exemple rue Saint Denys, à l'Hôtel du Grand Cerf), même si des relais existent très près de celui-ci (la Muette, Porte des Princes, Saint Denys...). Pour donner une idée de son importance le relais de Paris compte 135 chevaux en 1756, et 229 en 1849. Il reste nécessaire de décharger les bagages pour passer d'une route de Poste aux Chevaux à une autre, mais on est là comme dans la situation du changement de train dans une gare. Il n'y a pas interconnexion pure puisque ce n'est pas la même malle-poste qui va de Lille à Bordeaux en passant par Paris, mais qu'il s'agit de deux trajets clairement séparés. Il n'empêche, on a là une interconnexion réelle dans système de voies de transport à l'échelle de la France. Ajoutons par ailleurs que l'idée de connectivité est précocement présente dans le cas de la Poste aux Chevaux. Elle l'est d'abord, de façon évidente dans la pratique de l'époque puisque lorsqu'un chemin entre deux lieux est mauvais, les acteurs du XVIIIe siècle utilisent le "chevelu des routes". C'est une époque où, comme l'écrit Franck Imberdis dans sa thèse sur les routes d'Auvergne, le réseau routier français est constitué d'un ensemble de chemins de terre, "tous sensiblement équivalents, et dont certains ne sont des routes que parce que le trafic à longue distance les emprunte de préférence aux autres. Parfois, selon l'état du sol et les nécessités du moment, les relations commerciales abandonnent telle voie pour telle autre ; souvent aussi, elles se partagent simultanément deux trajets parallèles"30.

La connectivité est par ailleurs de plus en plus présente dans la façon de présenter les routes à l'intérieur des Livres de Poste. En 1708, la seule alternative proposée correspond à l'itinéraire Paris-Lyon. Dans le premier cas, on passe par Nevers, Moulin, puis Roanne, dans le second, on passe par Auxerre, Dijon et Macon. En 1758, on trouve 2 Paris-Lyon (très proches des itinéraires de 1708), 2 Paris-Angers, 2 Paris-Rennes, 2 Paris-Caen et 2 AmiensLille. Enfin, en 1810, et là, la liste n'est pas exhaustive, on trouve 3 Paris-Anvers, 3 ParisLyon, 2 Paris-Basle, 2 Paris-Bordeaux, 2 Paris-Lille, 2 Paris-Strasbourg, 2 Paris-Toulon... Dès 1708 , la différence entre les itinéraires proposés est rendue visible par le nombre de «postes », entendons d'unité de mesure des distances et des coûts. Ainsi, en 1758 on peut comparer la "Route de Paris à Lyon par le Bourbonnois : 65 postes", à la "Route de Paris à Lyon par la Bourgogne : 59 postes”. En 1810, la différence entre les trois routes de Paris à Lyon (par Melun, Auxerre et Autun 59 postes 1/4; par Nevers et Moulin 58 poste 1/2; par

\footnotetext{
${ }^{30}$ IMBERDIS, Franck, Le réseau routier de l'Auvergne au XVIIIe siècle, ses origines et son évolution, Paris, Presses Universitaires de France, 1967, p.324.
} 
Troyes, Dijon et Macon 61 poste $1 / 2$ ) se réduit à 3 postes. Il n'en reste pas moins que la connectivité va croissant dans le système de communication de la Poste aux Chevaux pendant la période.

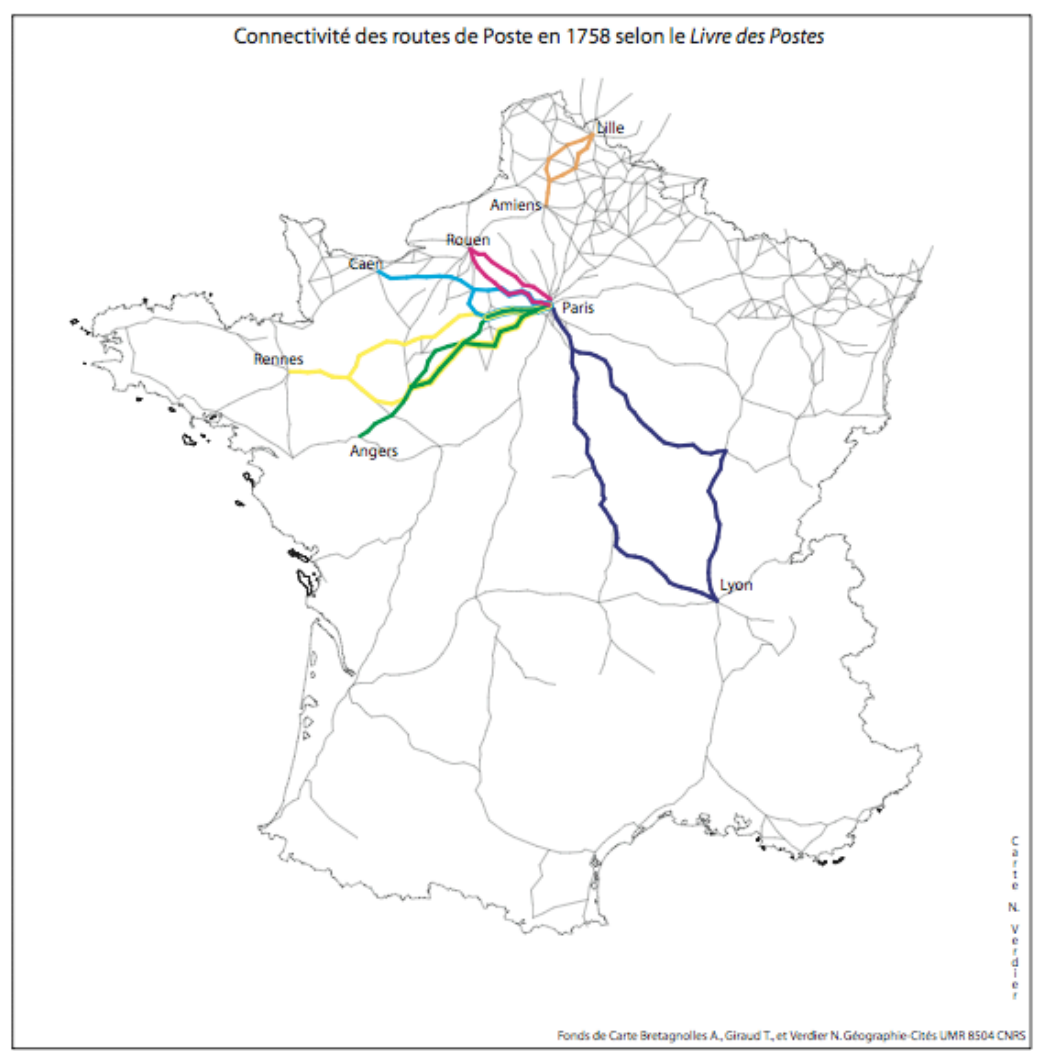

Reste la question de la hiérarchie, vue au milieu des années 1980 comme étant nécessaire au réseau. On sait que l'usage du mot "hiérarchie" reste longtemps limité au seul domaine de la théologie, même s'il gagne très progressivement un domaine plus vaste à partir du XVIIIe siècle, et surtout en fait après le deuxième tiers du XIXe siècle. Mais des pratiques de la hiérarchisation se mettent place bien avant et concernent par exemple les villes, classées en fonction de leur population dès le XVIIIe siècle, par exemple, dans les travaux de Hesse $^{31}{ }^{31}$. Pour les routes de Poste, cette hiérarchie apparaît clairement dans les Livres de Postes. Mais ces livres varient dans leur forme durant la période, ce qui se reflète dans leur description de la hiérarchie. En 1708 le classement est géographique et centré sur Paris, il décrit les axes dans le sens des aiguilles d'une montre : Paris-Lyon-Marseille, suivi de Paris Toulouse Narbonne, suivit de Paris Bordeaux Bayonne, puis de Paris Angers, etc. Mais, cet ordre général s'accompagne d'une hiérarchisation qui place d'abord la route de Paris à une

\footnotetext{
${ }^{31}$ Verdier, N., "Hierarchy, a short history of a world in Western thought", in Pumain D. (ed.), Hierarchy in Natural and Social Sciences, Dordrecht, Springer, 2005, pp. 13-37.
} 
grande ville, puis de cette grande ville à une autre ville, puis de deux villes non directement liées à Paris, entre elles, puis sont décrites les "communications", et non plus les routes, et pour finir en 1708 , les "traverses".

\section{La hiérarchie des tracés}
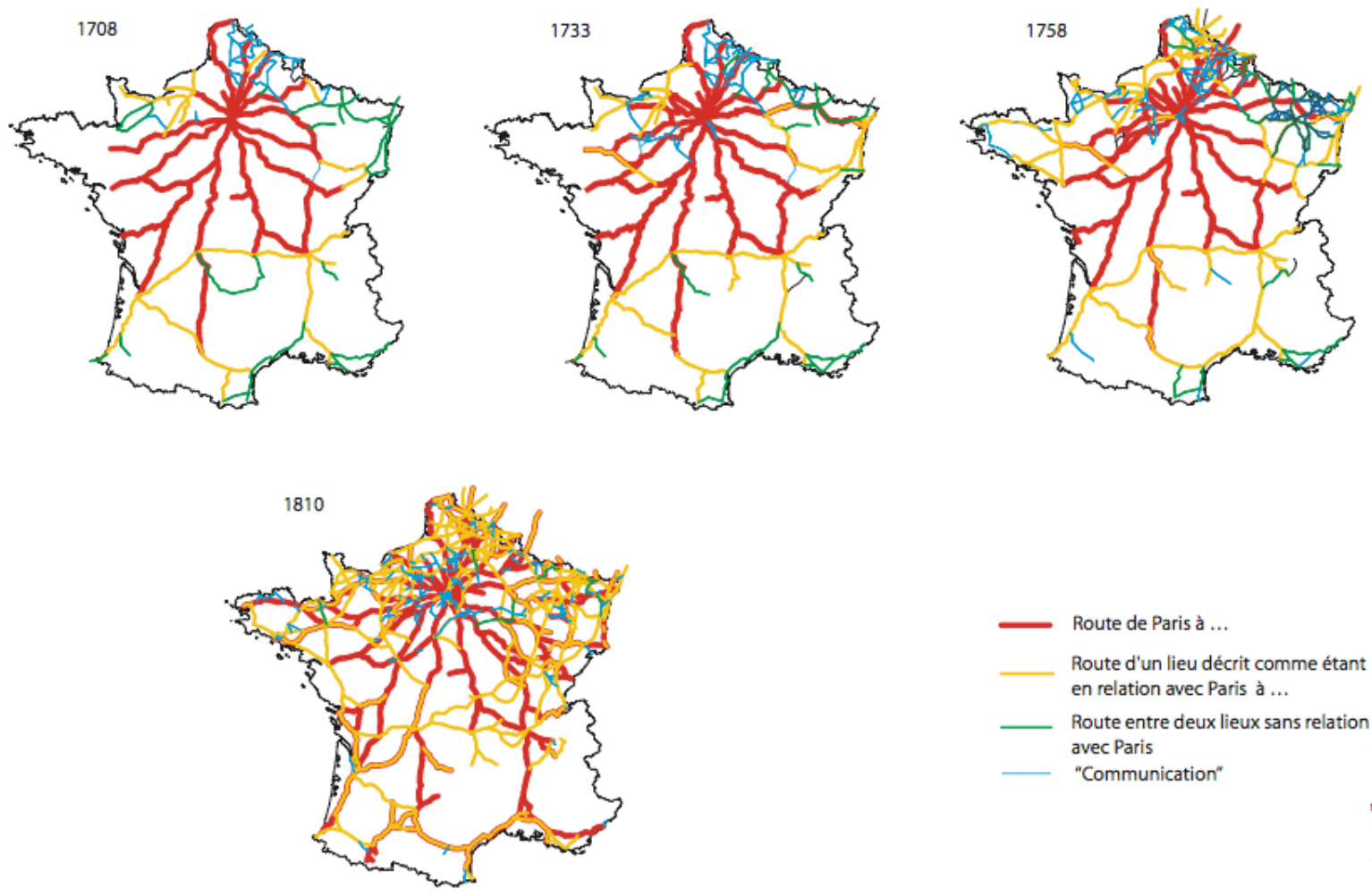

Route de Paris à ...
Route d'un lieu décrit comme étant en relation avec Paris à ...

Route entre deux lieux sans relation avec Paris

"Communication"

Nous sommes là très proche de la classification qui sera prônée 30 ans plus tard par Orry. En 1758, devant la complexité croissante apparaît une "Table alphabétique de toutes les routes de Postes de France, traverses et communications contenües en ce livre", y sont toujours distingues, routes, communications et traverses. La grande évolution apparaît vers 1774 , et classe les routes par ordre alphabétique. Dès lors, les premières routes ne partent pas de Paris. En revanche, les premières pages proposent, par exception l'ensemble des communications autour de Paris et de Versailles. La catégorie "traverse" disparaît, mais les routes et communications se maintiennent. En 1810, l'ordre alphabétique est conservé, et les communications de Paris et de Versailles trouvent à l'intérieur de livre leur ordre logique. Les routes sont numérotées (91 routes). La catégorie "communication" est toujours présente ; elle augmente en quantité dans le Livre... Autrement dit, il existe une hiérarchisation, qui d'abord exprimée, en tenant compte d'un ordre géographique, passe au croisement de la hiérarchie 
routes-communications articulées à un ordre alphabétique. Dans ces derniers cas (ceux de 1783 et de 1810), une deuxième hiérarchie s'impose puisque si l'ordre alphabétique et à peu près bien respectée, dans les faits, la très grande majorité des routes partent de Paris, et non d'une autre ville (profitons-en pour évoquer la route $\mathrm{n}^{\circ} 89$, de Strasbourg à Bordeaux par Lyon qui offre une transversale qui déroge à la norme en ne passant pas par Paris).

\section{Le réseau : un concept utilisé au XVIIIe siècle.}

Toute recherche d'ancêtre au sein d'une discipline ou à l'intérieur d'un thème de recherche est une tentative pour mieux appréhender l'origine des concepts qui y sont employés. Les quelques résultats de cette enquête montrent que les problématiques du réseau existaient bien avant les années 1820 , et que si des conceptualisations très proches peuvent en être trouvées une quarantaine d'années plus tôt, ce qui bien des enquêtes avaient démontré dans les années 1980, dans les faits, il est possible de trouver les prémices de ces concepts dès la fin du XVIIe siècle. L'observation d'un corpus étendu de cartes et de tableaux, liés à la Poste aux chevaux, au Génie, aux Ponts et Chaussées, voire à la Poste aux lettres montre clairement une description graphique de réseaux physiques qui mènent à des conceptualisations, soit par la hiérarchisation, soit par la production d'itinéraires. L'usage d'autres mots comme celui d'itinéraire, montre que, en l'absence du mot réseau, d'autres peuvent porter le concept émergeant. Les productions à diverses échelles montrent que le concept se retrouve à de très nombreux niveaux. Enfin, le cas de la Poste aux chevaux montre qu'une hiérarchisation des voies, comprises à l'intérieur d'un système de communications apparaît dès le début du XVIIIe siècle. Tous les éléments minimums du réseau existent donc bien avant les années 1820. Si l'articulation de tous ces principes n'est pas toujours effectuée, la Poste aux chevaux donne l'exemple de cette conceptualisation précoce.

Cette période, d'avant le mot, peut être vu comme un laboratoire où toutes les expériences sont bonnes à faire, autrement dit où tous les choix principaux n'ont pas encore été faits, et où des certitudes ne se sont pas imposées. Prenons-en un dernier exemple qui montrera combien une recherche d'antériorité renouvelée peut enrichir nos problématiques. Il semble aujourd'hui relativement évident que les réseaux techniques croissent par un phénomène simple d'ajouts successifs. Les lignes de chemin de fer voire les systèmes d'adduction d'eau correspondent en apparence à cette forme de croissance. Rien là de bien extraordinaire en apparence. Mais à y regarder de plus près, dans le cadre de la Poste aux 
Chevaux, que se passe t'il ? Dans les faits, la croissance du réseau s'accompagne d'intenses fluctuations dans le cadre des routes qui semblaient acquises.

\section{Evolution du réseau des routes postales sur la période 1758 - 1783}

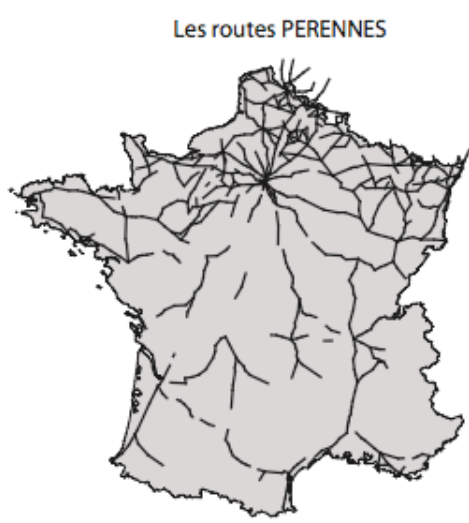

- Route postale en 1758 et 1783
Les routes APPARUES

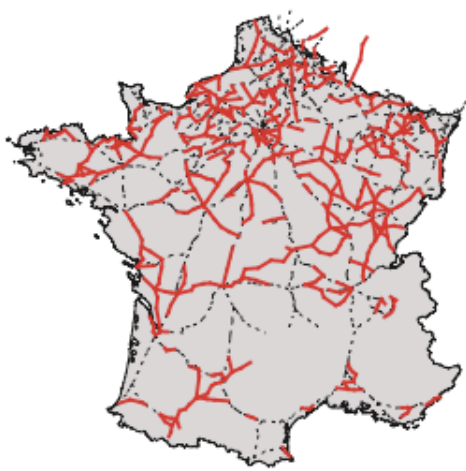

$\begin{array}{cc}\text { - } & \text { Route postale en } 1783 \\ \text { - - Résezu postal en } 1758\end{array}$
Les routes DISPARUES

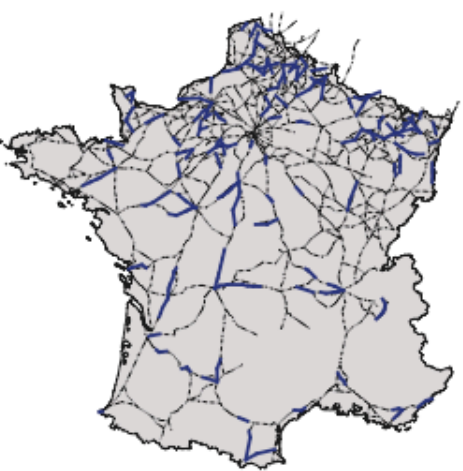

- Route postale en 1758

Ce qui apparaît ici, c'est que si de nombreuses routes se maintiennent d'une date à une autre, dans les faits, de nombreuses routes apparaissent là où d'autres existaient à peu de choses près. Autrement dit, la croissance s'accompagne d'un retravaille constant ${ }^{32}$. Ceux qui sont amenés à étudier les réseaux connaissent de nombreux cas similaires, mais ce qui est important ici réside dans l'ampleur du processus. Les modalités de fonctionnement du réseau de la poste aux chevaux sont donc très proches du P2P que beaucoup d'entre nous avons considéré comme une véritable révolution dans l'usage des réseaux. Une institution comme la Poste aux chevaux a donc été capable de gérer au jour le jour ces apparitions-disparitions, en procédant par des dérivations qui permettaient à l'ensemble du système de se maintenir.

\footnotetext{
${ }^{32}$ Bretagnolles, A. et Verdier N., "L'extension du réseau des routes de Poste en France, 1708-1833”, Actes (à paraître en août 2006) du colloque Les réseaux postaux en Europe du XVIIle au XXIe siècles, construction et développement, fonctions et modèles, Paris, Comité pour l'histoire de la Poste, 10-12 juin 2004.
} 